\title{
RESOLUCIÓN DE LAS ECUACIONES EN DIFERENCIAS
}

\section{SOLVING DIFFERENCE EQUATIONS}

Arturo Pérez París: Universidad de Alcalá. Madrid (España)

arturo.perez@mixmail.com

Julio Gutiérrez Muñoz: Universidad de Alcalá. Madrid (España) julio.gutierrez@uah.es

\section{CURRÍCULUM VITAE}

Escuela Politécnica de la Universidad de Alcalá de Henares. Ingeniero electrónico y literato. Destacan sus estudios sobre aplicaciones eléctricas y motricidad sobre los que ha publicado varios artículos científicos.

Catedrático de Física Atómica, Molecular y Nuclear de la Escuela Politécnica Superior de la Universidad de Alcalá de Henares (Madrid). En la actualidad imparte clases de la asignatura de Física incluida en la titulación de Grado en Química de dicha Universidad. Autor de numerosos artículos científicos. Miembro fundador de GRUA (Grupo de Reflexión de la Universidad de Alcalá de Henares). Vicerrector de la Universidad de Alcalá de Henares. Director de la Revista Vivat Academia.

\section{RESUMEN}

En este artículo les presentamos una breve exposición de la resolución de ecuaciones en diferencias por el método del polinomio característico, también llamado del anulador. En el artículo anterior (Vivat Academia n 31, diciembre 2001- enero 2002) 
hicimos una exposición del planteamiento general de las ecuaciones en diferencias, con el estudio de los criterios de estabilidad y asintoticidad, y dimos una serie de pautas para poder manipularlas adecuadamente. Vimos que cualquier ecuación de este tipo puede ser resuelta por el método estándar de la recurrencia, establecido en el primer artículo introductorio, denominado las "Torres de Hanoi" (Vivat Academia n 30, noviembre 2001). En los casos más simples ello puede ser útil, sin embargo, en la generalidad de los casos debemos recurrir a otros métodos con el fin de asegurarnos la obtención de las soluciones, sin hacer uso de complejos y tediosos cálculos. En este artículo mostraremos uno de estos métodos de resolución.

\section{PALABRAS CLAVE}

Ecuaciones - Sistemas - Método del polinomio - Coeficiente

\section{ABSTRACT}

In this paper we present a brief summary of the resolution of difference equations by the method of the characteristic polynomial, also called the annihilator. In the previous article (Vivat Academia No. 31, December 2001 - January 2002) made a presentation of the general approach of the difference equations, with the study of the stability criteria and asymptotic, and we took a number of guidelines to handle them properly. We saw that any such equation can be solved by the standard method of recurrence, established in the first introductory article, called the Towers of Hanoi (Vivat Academia No. 30, November 2001). In the simplest cases it may be useful, however, the majority of cases we must resort to other methods to ensure obtaining the solutions, without using complex and tedious calculations. In this article we show one of these methods of resolution. 


\section{KEY WORDS}

Equations - Systems - Polynomial method - Coefficient

\section{ÍNDICE}

\section{Introducción}

2. Soluciones de las ecuaciones en diferencias lineales no homogéneas de orden $\mathrm{k}$

3. Teorema de existencia y unicidad para valores iniciales

4. Métodos de resolución abreviados de las ecuaciones en diferencias: El polinomio característico

4.1. Ecuaciones de primer orden con coeficientes constantes

4.2. Caso de las ecuaciones inhomogéneas con coeficientes constantes

4.3. Uso del método del anulador o polinomio característico para ecuaciones inhomogéneas

5. Ecuaciones de orden superior con coeficientes constantes

6. Generalización del método del polinomio característico en la búsqueda de soluciones de una ecuación inhomogénea con coeficientes no constantes

7. Resumen del método del polinomio característico

8. Conclusión

\section{TEXTO:}

\section{Introducción}

En el artículo anterior (Vivat Academia $n^{\circ} 31$, diciembre 2001- enero 2002) hicimos una exposición del planteamiento general de las ecuaciones en diferencias, con el 
estudio de los criterios de estabilidad y asintoticidad, y dimos una serie de pautas para poder manipularlas adecuadamente. Vimos que cualquier ecuación de este tipo puede ser resuelta por el método estándar de la recurrencia, establecido en el primer artículo introductorio, denominado las "Torres de Hanoi" (Vivat Academia n ${ }^{\text {o 30, }}$ noviembre 2001). En los casos más simples ello puede ser útil, sin embargo, en la generalidad de los casos debemos recurrir a otros métodos con el fin de asegurarnos la obtención de las soluciones, sin hacer uso de complejos y tediosos cálculos. En este artículo mostraremos uno de estos métodos de resolución.

Asimismo, al finalizar el artículo anterior mencionado, vimos la posibilidad de obtener soluciones generales para las ecuaciones en diferencias. Expondremos a continuación el teorema de existencia y unicidad de las soluciones.

\section{Soluciones de las ecuaciones en diferencias lineales no homogéneas de orden $k$}

Sea la ecuación en diferencias, lineal, no homogénea, de orden k, con coeficientes no constantes

$$
y[n+1]=\sum_{k=0}^{n} g_{k}(k) y[k]+r[n]
$$

Esta ecuación también se puede escribir en la forma

$y[n+k+1]=\sum_{j=0}^{k} g_{n+j}(n+j) y[n+j]+r[n]$

donde se ve claramente el orden de la ecuación. Los g[i] y r[n] son los coeficientes no constantes, determinando el último la inhomogeneidad de la ecuación. El término $\mathrm{r}[\mathrm{n}]$ es llamado término de forzamiento, fuerza externa, control o entrada ("input") 
del sistema, ya que se corresponde, en la mayoría de los problemas físicos, con una fuerza externa que obliga al sistema a comportarse de una forma determinada.

Para encontrar la solución necesitamos de k valores que fijen las condiciones iniciales del problema.

La ecuación homogénea asociada, a la que no imponemos condiciones iniciales, será $y[n+k+1]=\sum_{j=0}^{k} g_{n+j}(n+j) y[n+j]$

Sea $u[n]$ una solución general de esta ecuación, es decir, una solución válida para cualquier condición inicial. Sea z[n] una solución particular de la ecuación completa, válida solamente para el conjunto de condiciones iniciales

$y[n]=a 0, y[n+1]=a 1, y[n+2]=a 2, \ldots, y[n+k]=a k$

La suma

$\mathbf{w}[\mathbf{n}]=\mathbf{u}[\mathbf{n}]+\mathrm{z}[\mathrm{n}]$

es también solución de la ecuación completa, ya que será z[n] la que determine la igualdad de la ecuación con $\mathrm{r}[\mathrm{n}]$ y la coincidencia con los valores iniciales.

Como es fácil de ver, si z1[n] y z2[n] son dos soluciones de la ecuación completa, su diferencia

$\mathbf{z} 1[n]-\mathbf{z}[\mathbf{n}]$

será solución de la ecuación homogénea asociada.

Sean u1(n) y u2(n) dos soluciones generales de la ecuación homogénea, es decir dos soluciones válidas, para cualquier valor inicial. Entonces, tal como concluíamos al 
finalizar el artículo anterior, una combinación lineal de estas dos soluciones será también solución de la ecuación homogénea

\section{$C \mathfrak{u} 1[n]+D \mathfrak{u} 2[n]$}

con C y D dos valores reales cualesquiera.

Queda por determinar si la solución w[n], suma de la solución general de la homogénea y una solución particular de la ecuación completa, que satisface además el conjunto de valores iniciales, es única.

\section{Teorema de existencia y unicidad para valores iniciales}

Como habíamos concluido en el artículo anterior, si w1[n] y w2[n] son dos soluciones de la ecuación completa que satisfacen el conjunto de condiciones iniciales, también una combinación de ellas

\section{$A w 1[n]+B w 2[n]$}

debe ser solución. Sin embargo, la combinación no es arbitraria. Sustituyendo la suma anterior en la ecuación completa, observamos que debe cumplirse la condición $\mathrm{A}+\mathrm{B}=1$.

Así pues, veamos si la existencia de estas dos soluciones diferentes w1 y w2 está permitida o, por el contrario, ambas deben coincidir.

Tengamos

$W[n]=A w 1[n]+B w 2[n]$

que, a su vez, siendo solución de la ecuación completa, será de la forma

$\mathbf{W}[\mathbf{n}]=\mathrm{U}[\mathbf{n}]+\mathrm{Z}[\mathrm{n}]$ 
con

$w 1[n]=u 1[n]+z 1[n] ; w 2[n]=u 2[n]+z 2[n]$

Como cualquier combinación lineal de soluciones de la ecuación homogénea también lo es

$\mathrm{U}[\mathrm{n}]=\mathrm{A} \mathbf{u} 1[\mathrm{n}]+\mathbf{B} \mathbf{u}[\mathrm{n}]$

es solución de la ecuación homogénea. Podemos tomar, entonces U[n] como la solución general, donde están incluidas todas las soluciones posibles de la ecuación homogénea. Queda por ver si

$\mathrm{Z}[\mathrm{n}]=\mathbf{A} \mathbf{z} 1[\mathrm{n}]+\mathbf{B} \mathbf{z} 2[\mathrm{n}]$,

es solución de la ecuación completa, con la condición A+B=1.

Tomemos primeramente $\mathrm{A}=1$ - $\mathrm{B}$, entonces

$Z[n]=(1-B) z 1[n]+B z 2[n]=$

$=-B(z 1[n]-z 2[n])+z 1[n]$

Tomemos ahora $\mathrm{B}=1-\mathrm{A}$, entonces

$\mathrm{Z}[\mathrm{n}]=(\mathbf{1}-\mathrm{A}) \mathrm{z} 2[\mathrm{n}]+\mathrm{A} \mathrm{z} 1[\mathrm{n}]=$

$=A(z 1[n]-z 2[n])+z 2[n]$

Anteriormente hemos visto que (z1[n] - z2[n]) es solución de la ecuación homogénea y no de la completa. Por otra parte como solución particular de la completa nos queda

$\mathrm{Z}[\mathrm{n}]=\mathbf{z} 1[\mathrm{n}] \mathrm{y}$ 
$\mathrm{Z}[\mathrm{n}]=\mathrm{z} 2[\mathrm{n}]$

Es decir, la solución particular es única por lo que respecta a la ecuación completa, ya que (z1[n] - z2[n]) al ser una solución de la ecuación homogñenea debería estar ya inluida en la solución general U[n] (según lo anterior esa solución es idénticamente nula).

Quedaría todavía una cuestión por contestar, correspondiente a la existencia de la solución. Es decir, dada una ecuación inhomogenea, lineal, ¿existe siempre solución? Acabamos de ver que, si existe, la solución es única. Pero la prueba de existencia no es simple, salvo que la ecuación sea homogénea o inhomogénea con coeficientes constantes. Para este curso introductorio, sería demasiado tedioso comprobar el teorema de existencia, por lo que dejamos al lector el trabajo de buscar en la bibliografía las pruebas pertinentes.

\section{Métodos de resolución abreviados de las ecuaciones en diferencias: El polinomio característico}

El método de la recurrencia, como ya hemos mencionado, es una forma bastante tediosa y larga de encontrar las soluciones a las ecuaciones en diferencias. En muchos de los casos existen formas más abreviadas de resolver este tipo de ecuaciones. Mostramos aquí una de ellas, sobre todo porque guarda estrecha relación con los métodos de resolución de las ecuaciones diferenciales, que es lo que en suma nos importa, ya que pretendemos establecer un paralelismo entre ambos tipos de ecuaciones, a fin de establecer el método de resolución numérico llamado de las diferencias finitas. 


\subsection{Ecuaciones de primer orden con coeficientes constantes}

Para entender mejor el proceso, partamos de una sencilla ecuación homogénea en diferencias, con coeficientes constantes

$\mathbf{y}[\mathbf{n}+1]-\mathrm{b} \mathbf{y}[\mathrm{n}]=0$

Utilizando la nomenclatura de operadores desarrollada en el artículo anterior, esta ecuación se puede rescribir en la forma

$(\mathrm{E}-\mathrm{b}$ I) $\mathrm{y}[\mathrm{n}]=\mathbf{0}$

siendo E el operador desplazamiento, también llamado salto o cambio, e I el operador identidad

$\mathrm{E}=$ Operador de cambio: $\mathbf{E}(\mathbf{u}[\mathbf{n}])=\mathbf{u}[\mathbf{n}+\mathbf{1}]$

$\mathrm{I}=$ Operador identidad: $\mathbf{I}(\mathbf{u}[\mathbf{n}])=\mathbf{u}[\mathbf{n}]$

Usando la recurrencia, y suponiendo la condición inicial y $[0]=\mathrm{A}$

$\mathrm{y}[\mathrm{n}+1]=\mathrm{b} \mathbf{y}[\mathrm{n}]$

$y[1]=b y[0]=b \cdot A$

$y[2]=b \quad y[1]=b 2 \cdot A \ldots$

$\mathrm{y}[\mathrm{n}]=\mathrm{b} \mathbf{y}[\mathrm{n}-1]=\mathrm{bn} \cdot \mathrm{A}$

En términos de operadores, tendremos:

$y[1]=E y[0]=b I \cdot A$

$y[2]=E y[1]=E 2 y[0]=b 2 \cdot I 2 A \ldots$

$y[n]=E y[n-1]=E n y[0]=b n \cdot \operatorname{In} A$ 
También podríamos expresar la solución de la ecuación en la forma:

En $y[0]-b n \cdot I n A=0$

$\{$ En - bn In $\} y[0]=0$

Es decir, podemos inferir que el operador En toma como valor bn In, o equivalentemente, el operador $\mathrm{E}$ es exactamente b I. Nótese que ello equivale a anular el paréntesis (E - b I) en la expresión de partida que define la ecuación en diferencias que nos ocupa:

$(\mathrm{E}-\mathrm{b} \mathrm{I}) \mathrm{y}[\mathrm{n}]=0$

Este paréntesis es denominado polinomio característico.

$p(E)=E-b I$

$\mathrm{Su}$ resolución, equivalente a encontrar los valores del operador E que anulan dicho paréntesis, nos da la solución de la ecuación en diferencias, (bien es verdad que en este sencillo caso de ecuación homogénea). Así:

$y[n]=E y[n-1]=E n y[0]=E n A=b n A$

\subsection{Caso de las ecuaciones inhomogéneas con coeficientes constantes}

Veamos qué ocurre para una ecuación en diferencias inhomogénea, con coeficientes constantes, tal como:

$y[n+1]-b y[n]=c$

con la condición inicial y[0] = A. En términos de operadores:

$(\mathrm{E}-\mathrm{b}$ I) $\mathrm{y}[\mathrm{n}]=\mathrm{c}$ 
En este caso la recurrencia nos proporciona:

$$
\begin{aligned}
& y[1]=b y[0]+c=b \cdot A+c \\
& y[2]=b y[1]+c=b 2 \cdot A+b c+c \\
& y[3]=b y[2]+c=b 3 \cdot A+b 2 c+b c+c \ldots \\
& y[n]=b y[n-1]+c= \\
& b n \cdot A+\{b(n-1)+b(n-2)+\ldots+b 2+b+1\} c \\
& y[n+1]=b y[n]+c= \\
& b[b n \cdot A+\{b(n-1) c+b(n-2)+\ldots+b 2+b+1\} c]+c= \\
& y[n+1]=b \cdot y[n]+c=b^{(n+1)} y[0]+\sum_{k=0}^{n} b^{k} \cdot c
\end{aligned}
$$

El sumatorio de la derecha de la expresión anterior corresponde a la suma de los términos de una progresión geométrica de razón b. Recordemos el valor de esta suma, dada en el artículo "Torres de Hanoi":

$$
\begin{aligned}
& S n=a 1+a 2+\ldots \ldots \ldots \ldots+\text { an } \\
& r \cdot S n=a 2+a 3+\ldots \ldots+a n+a n \cdot r \\
& S n-r \cdot S n=a 1-a n r=> \\
& (1-r) S n=a 1-a n r=> \\
& S n=\frac{a_{1}-a_{n} \cdot r}{1-r}
\end{aligned}
$$


En consecuencia,

$$
y[n+1]=b \cdot y[n]+c=b^{(n+1)} y[0]+\left(\frac{b b^{n}-1}{b-1}\right) c
$$

\section{o también}

$$
y[n]=b^{n} y[0]+\left(\frac{b^{n}-1}{b-1}\right) c
$$

Para conseguir un método abreviado, podemos optar por otro procedimiento, en un intento de utilizar el método del polinomio característico (método del anulador), descrito para la solución de la ecuación homogénea. Sin embargo, primeramente vamos a describir una forma sencilla, sólo útil para las ecuaciones inhomogéneas en coeficientes constantes.

Si pudiéramos reducir la ecuación inhomogénea que nos ocupa a una ecuación homogénea, de la que sabemos encontrar la solución por el método del polinomio característico, habríamos resuelto el problema. De esa forma, la solución más general de la ecuación anterior sería la solución general de una ecuación homogénea a la que hemos obligado a obedecer la condición inicial. Transformemos la ecuación en una nueva, redefiniendo los estados en esta forma

$y p[n]=F y[n]+G c$

El siguiente estado será

$y p[n+1]=F y[n+1]+G c$

De las expresiones anteriores encontramos

$(y p[n+1]-G$ c) F-1 = y[n+1]

$(y p[n]-G c) F-1=y[n]$ 
Las constantes F y G se determinan de forma que la ecuación resultante para yp[n] sea homogénea. Sustituyendo los valores anteriores en la ecuación inhomogénea de partida, encontramos

$F=G(b-1)$

Y la solución de la ecuación resultante

$\operatorname{yp}[n+1]-b$ yp $[n]=0$

puede encontrarse por el método del anulador del polinomio característico

$(\mathrm{E}-\mathrm{b}$ I) yp[n] $=0$

Es decir

$y p[n]=\operatorname{Eyp}[n-1]=\operatorname{En} y p[0]=$ bn yp[0]

Ahora debemos identificar las condiciones iniciales de yp[n] en función de las de y[n]. De la transformación

$y p[0]=F y[0]+G c$

obtenemos

$\mathrm{yp}[\mathrm{n}]=$ bn $(\mathrm{F} \mathrm{y}[0]+\mathrm{G} \mathrm{c})$

donde, sustituyendo el valor de yp[n], resulta

$F y[n]+G c=b n(F y[0]+G c)$

$y[n]=b n(y[0]+c G / F)-c$ G/F

De las condiciones sobre F y G determinamos la solución final de la ecuación en diferencias inhomogénea motivo de estudio 


$$
y[n]=b^{n} y[0]+\left(\frac{b^{n}-1}{b-1}\right) c
$$

\subsection{Uso del método del anulador o polinomio característico para ecuaciones inhomogéneas}

Observemos que la ecuación puede ser escrita de la forma:

$(E-b I) y[n]-c=0$

En ella, tomando el anulador de la ecuación homogénea

$(\mathrm{E} 1-\mathrm{b}$ I) $\mathrm{yh}[\mathrm{n}]=0$

obtendríamos sus soluciones. En este caso

$\mathbf{E} 1=\mathbf{b} \mathbf{I}$

equivalentemente

$\operatorname{yh}[\mathrm{n}]=\mathrm{A}$ bn

con A una constante arbitraria. Nos faltaría ahora encontrar una solución particular de la ecuación completa.

Tengamos en cuenta que el operador diferencia actuando sobre una constante es cero, es decir:

$D c=0$

De la definición de operador cambio, $\mathrm{E}=(\mathrm{D}+\mathrm{I})$ :

$\mathrm{E} c=c ;(E-I) c=0$ 
Es decir, el anulador de c es (E - I). Consecuentemente, la ecuación

$(E-b$ I) $y[n]-c=0$

tiene una solución en términos de anuladores, consistente en encontrar el anulador de c, que llamaremos E2 y verifica

$(\mathrm{E} 2-\mathrm{I})(\mathrm{E}-\mathrm{b} \mathrm{I}) \mathrm{yp}[\mathrm{n}]=0=(\mathrm{E} 2-\mathrm{I}) \mathrm{c}$

siendo yp[n] una solución particular de la ecuación inhomogénea completa. La ecuación para yp[n] tiene dos soluciones, una usando el anulador (E - b I) y la otra usando (E2 - I), que son respectivamente, en términos del polinomio característico

$E=b$ I y $E 2=1$ I

es decir,

$\operatorname{yp} 1[n]=$ bn e $\operatorname{yp} 2[n]=1 n$

Pero aquí debemos tener en cuenta que la solución de las ecuaciones inhomogéneas la buscamos como suma de la ecuación general de la homogénea asociada y una solución particular de la inhomogénea completa. Con ello queremos indicar que la solución que obtengamos haciendo uso del polinomio

$(\mathrm{E}-\mathrm{b} \mathrm{I})=0$

es una solución de la ecuación homogénea, ya incluida en la resolución correspondiente de la ecuación asociada. Usar el anterior anulador nos proporcionaría una redundancia innecesaria. Así pues, sólo es necesario determinar la solución del polinomio

$(\mathrm{E} 2-\mathrm{I})=0$ 
Notemos que, en términos de operadores, la ecuación inhomogénea de partida se escribe como

\section{[Polinomio de $\mathrm{E}] \times \mathrm{y}[\mathrm{n}]=\mathrm{c}$}

y la solución particular se encuentra como el anulador de c, es decir

\section{[anulador $\mathrm{c}] \times$ [polinomio de $\mathrm{E}]=0$}

Consecuentemente la solución será la suma de la solución general correspondiente a la ecuación homogénea más la solución particular de la inhomogénea completa, encontrada utilizando el anulador precedente

E2 = I

que, en este caso, indica una solución

$\operatorname{yp} 2[n]=$ constante arbitraria $=B$

Así pues la solución buscada será:

$y[n]=y h[n]+y p 2[n]=A b n+B$

siendo A y B dos constantes arbitrarias, a determinar por medio de la propia ecuación y de la condición inicial.

Sustituyendo esta solución en la ecuación original

$y[n+1]-b y[n]=c$

o en forma equivalente

$(\mathrm{E} 1-\mathrm{b}$ I) $\mathrm{y}[\mathrm{n}]=\mathrm{c}$

es decir, 
$(\mathrm{E} 1-\mathrm{b}$ I) $(\mathrm{A}$ bn $+\mathrm{B})=\mathrm{c}$

Recordemos que la solución A bn lo es de la ecuación homogénea, luego la aplicación del anulador al primer término es cero. Por tanto, obtenemos

$(\mathrm{E} 1-\mathrm{b}$ I) $\mathrm{B}=\mathrm{c}$

Como el operador salto actuando sobre una constante la deja invariante, resulta

$B-b B=c=>B=c /(1-b)$

Utilicemos ahora la condición inicial que, en términos de la solución encontrada, será

$y[0]=A+c /(1-b)$

para obtener el valor de A

$A=y[0]-c /(1-b)=y[0]+c /(b-1)$

Luego la solución se escribirá

$y[n]=y[0] b n-c b n /(1-b)+c /(1-b)=$

$=y[0] b n+c(b n-1) /(b-1)$

Nótese que esta solución coincide exactamente con las encontradas por el método de la recurrencia y de la reducción a una homogénea. Debemos tener cuidado con la redundancia posible en soluciones de la ecuación homogénea asociada al buscar las soluciones particulares. 


\section{Ecuaciones de orden superior con coeficientes constantes}

Para abordar este apartado resolveremos unas ecuaciones prototipo que nos servirán de base:

$y[n+2]+y[n]=0$

Homogénea de orden dos.

Si la reescribimos en función del operador de desplazamiento, resulta:

$(\mathrm{E} 2+1 \mathrm{I}) \mathrm{y}[\mathrm{n}]=0$

Podemos obtener el polinomio característico y sus correspondientes raíces (en este caso pertenecientes al cuerpo de los números complejos e imaginarias puras):

$P(r)=r 2+1=0$

Raíces: $\mathbf{r} \mathbf{1}=\mathbf{j}$

$$
\mathbf{r} 2=-\mathbf{j}
$$

Así la solución de esta ecuación homogénea es:

$y[n]=P j ~ n+Q(-j) n$

si aplicamos las reglas de Euler será:

$$
\begin{aligned}
& y[n]=P \cdot 1^{n}\left(\cos \frac{\pi}{2} n+j \cdot \operatorname{sen} \frac{\pi}{2} n\right)+Q \cdot 1^{n} \cdot\left(\cos \frac{-\pi}{2} n+j \cdot \operatorname{sen} \frac{-\pi}{2} n\right)= \\
&(P+Q) \cdot\left(\cos \frac{\pi}{2} n\right)+j \cdot(P-Q) \cdot\left(\operatorname{sen} \frac{\pi}{2} n\right)
\end{aligned}
$$


Debemos buscar las soluciones reales de esta ecuación en diferencias, para ello basta con escoger las constantes "P" y "Q" de tal manera que sean complejos conjugados, es decir:

$P=A 1+j B 1$

$=>P+Q=2 \cdot A 1=A ；$

$\mathrm{j}(\mathrm{P}-\mathrm{Q})=-2 \cdot \mathrm{B} 1=\mathrm{B}$;

donde

$Q=A 1-\mathbf{j} \cdot \mathbf{B} 1$

resultando la solución general:

$y[n]=A \cdot\left(\cos \frac{\pi}{2} n\right)+B \cdot\left(\operatorname{sen} \frac{\pi}{2} n\right)$

6. Generalización del método del polinomio característico en la búsqueda de soluciones de una ecuación inhomogénea con coeficientes no constantes

Sea la ecuación inhomogénea con coeficientes no constantes

$y[n+k+1]-\sum_{j=0}^{k} g_{n+j}(n+j) y[n+j]-r[n]=0$

Cualquier solución y[n] tomará la forma

$y[n]=\sum_{i=1}^{k} a_{i} h_{i}[n]+y_{p}[n]$ 
donde $\{h 1[n], h 2[n], h 3[n] \ldots h k[n]\}$ es un conjunto completo de soluciones de la ecuación homogénea asociada e yp[n] es una solución particular de la inhomogénea completa. Los coeficientes ai son constantes arbitrarias a determinar.

Sabemos usar el método del anulador para cualquier ecuación homogénea, centremos, por tanto, nuestra atención en la búsqueda de yp[n]. Debemos indicar que la utilización del método del anulador no es efectiva para cualquier expresión del término inhomogéneo r[n]. Se pueden encontrar reglas muy simples, sin embargo, para aquellos casos en que r[n] sea combinación lineal de funciones de la forma

bn, $\operatorname{sen}(b n), \cos (b n)$, nk

o productos de éstas, habituales en las ecuaciones de sistemas físicos.

Denominaremos operador anulador $\mathrm{N}(\mathrm{E})$ del término de forzamiento $\mathrm{r}[\mathrm{n}]$, donde $\mathrm{E}$ representa el operador cambio o salto, a un polinomio de E que anule el término de inhomogeneidad, es decir, verifique

$\mathbf{N}(\mathrm{E}) \mathbf{r}[\mathrm{n}]=\mathbf{0}$

Mediante la búsqueda de las raíces de este polinomio (soluciones del polinomio igualado a cero), encontraremos las soluciones particulares de la ecuación completa.

Recordemos que en términos de operadores la ecuación inhomogénea de partida se escribe como

\section{[Polinomio de $E] \times y[n]=$}

término de forzamiento

y la solución particular se encuentra como el anulador de la fuerza externa, es decir

[anulador del término de forzamiento] 
$x[$ polinomio de $\mathrm{E}]=0$

Pongamos unos ejemplos:

Ejemplo 1:

Si $\mathbf{r}[\mathbf{n}]=$ bn, entonces

$\mathrm{E}(\mathrm{bn})=\mathrm{bn}+\mathbf{1}$

consecuentemente

$(\mathrm{E}-\mathrm{b} \mathrm{I}) \mathrm{r}[\mathrm{n}]=0=>$

$(E-b$ I) yp[n] = 0 e yp[n] = bn

Ejemplo 2:

Si $r[n]=\cos (n p / 2)$, entonces

$E \cos (n p / 2)=\cos (p(n+1) / 2)=$

$=-\operatorname{sen}(n p / 2) y$

$E 2 \cos (n p / 2)=E[-\operatorname{sen}(n p / 2)]=$

$=-\operatorname{sen}(p(n+1) / 2)=-\cos (n p / 2)$,

consecuentemente

$(\mathrm{E} 2-\mathrm{I}) \mathrm{r}[\mathrm{n}]=0=>(\mathrm{E} 2+\mathrm{I}) \mathrm{yp}[\mathrm{n}]=0$

$\operatorname{eyp}[n]=\cos (n p / 2)$ 
Escribimos pues la ecuación de la forma:

$P(E) y[n]=r[n]$

y encontramos las soluciones haciendo

$N(E) P(E) y[n]=0$

es decir, obteniendo las raíces $11,12,13, \ldots, 1 \mathrm{k}$ del polinomio característico $\mathrm{P}(\mathrm{E})$ correspondientes a la ecuación homogénea

$\mathrm{P}(\mathrm{E}) \mathrm{y}[\mathrm{n}]=0$

La solución particular será

$\mathrm{N}(\mathrm{E}) \mathrm{yp}[\mathrm{n}]=0$

que se determina encontrando las raíces $m 1, m 2, m 3, \ldots, \mathrm{mj}$ del polinomio característico $\mathrm{N}(\mathrm{E})$, correspondientes al anulador de la fuerza externa.

Debemos ahora hacer un par de consideraciones.

\section{Caso 1:}

Ninguno de los coeficientes lk es igual a alguno de los coeficientes mj, entonces podemos escribir la solución particular yp[n] como la solución general de la ecuación $\mathrm{N}(\mathrm{E}) \mathrm{yp}[\mathrm{n}]=0$ con constantes, en principio, indeterminadas, a encontrar sustituyendo la solución en la ecuación inhomogénea de partida.

\section{Caso 2:}

Ocurre que $1 \mathrm{k}=\mathrm{mj}$ para algunos valores de estas constantes. Entonces el conjunto de raíces características de la ecuación $\mathrm{N}(\mathrm{E}) \mathrm{P}(\mathrm{E}) \mathrm{y}[\mathrm{n}]=0$, es la unión de los dos conjuntos $\{1 \mathrm{k}\}\{\mathrm{mj}\}$ y, por tanto, contiene raíces múltiples. Para determinar la solución particular 
yp[n], debemos desechar de las soluciones que proporciona $\mathrm{N}(\mathrm{E}) \mathrm{P}(\mathrm{E}) \mathrm{y}[\mathrm{n}]=0$ aquellas que ya están incluidas en la solución general de la ecuación homogénea asociada que, en otro caso, serían redundantes.

\section{Resumen del método del polinomio característico}

Hemos recogido los casos más básicos de resolución de este tipo de ecuaciones por el método del polinomio característico, que se puede extender a la búsqueda de soluciones particulares de las ecuaciones no homogéneas, incluso en coeficientes no constantes. De forma genérica, podría plantearse un método de trabajo que sintetizara, a modo de receta, todo lo aquí expuesto:

$1^{\circ}$.) Haremos que el coeficiente de término de mayor orden sea la unidad operando adecuadamente la ecuación en diferencias. Es decir, si el término de mayor orden aparece multiplicado por alguna constante o expresión conocida del orden n, debemos dividir toda la ecuación por dicha constante o expresión.

$2^{\circ}$.) Reescribiremos la ecuación en función del operador salto "E", recordando que:

$$
\mathrm{E}=\mathrm{y}[\mathbf{n}+\mathbf{1}]
$$

$\mathrm{E} 2=\mathrm{y}[\mathrm{n}+2]$

$3^{\circ}$.) Obtendremos el polinomio característico a partir de la homogénea asociada, y de él sus raíces, sacando así la solución general de la ecuación en diferencias. Éstas podrán ser, bien reales, con lo que la solución a la ecuación será de la forma:

raíz: 1

$=>1 \mathrm{n}, \mathrm{n} \cdot \mathrm{ln}, \mathrm{n} 2 \cdot \mathrm{ln} \ldots$ 
multiplicidad: $\mathrm{n}$

bien complejas, con lo cual la solución general sería:

raíz: $\mathbf{P}$ jn $+Q \cdot(-\mathbf{j}) \mathbf{n}$

$$
\begin{gathered}
=> \\
P \cdot 1^{n} \cdot\left(\cos \frac{\pi}{2} n+j \cdot \operatorname{sen} \frac{\pi}{2} n\right)+Q \cdot 1^{n} \cdot\left(\cos \frac{-\pi}{2} n+j \cdot \operatorname{sen} \frac{-\pi}{2} n\right)= \\
\\
(P+Q) \cdot\left(\cos \frac{\pi}{2} n\right)+j \cdot(P-Q) \cdot\left(\operatorname{sen} \frac{\pi}{2} n\right)
\end{gathered}
$$

\section{multiplicidad: 1}

si hubiera "n" multiplicidad, se seguiría la pauta marcada para para las soluciones reales.

$4^{\circ}$.) Si fuese preciso, porque la ecuación fuese no homogénea, a partir de la ecuación reescrita en "E", se igualaría al segundo término de la ecuación (la fuerza externa); buscaríamos su anulador (el correspondiente a este segundo término) y lo aplicaríamos a toda ella, obteniendo así una homogénea de orden superior.

$5^{\circ}$.) En caso de utilizar el método del anulador o polinomio característico para encontrar soluciones particulares de una ecuación inhomogénea, utilizando la suma de una solución particular de la completa y la solución general de la homogénea, debemos descartar la parte que coincida con la solución general obtenida para la homogénea asociada (esta parte resulta redundante).

$6^{\circ}$.) Igualaremos lo que queda de la solución, operada con la expresión en "E", de la ecuación original con el segundo término de dicha ecuación original, y con ello valoraremos los coeficientes arbitrarios de la solución particular. 
$7^{\circ}$.) Expondremos la solución final como suma de las soluciones general y particular.

\section{Conclusión}

En este artículo les hemos presentado una breve exposición de la resolución de ecuaciones en diferencias por el método del polinomio característico, también llamado del anulador. Hemos optado por una presentación particular, haciendo uso de ejemplos sencillos de fácil comprensión, para después llegar a la generalización del método, por ser una forma sencilla de mostrar la teoría de las ecuaciones en diferencias. Sin faltar al rigor matemático, hemos omitido muchos de los lemas, teoremas y definiciones, habituales en estos casos, para evitar la dispersión de la idea principal, así como el excesivo volumen del trabajo, que harían oscuro al neófito la sencillez de este método.

No hemos descrito otros posibles métodos, lo que dejamos para posibles trabajos futuros, porque es concretamente éste el que marca con más intensidad el paralelismo entre las ecuaciones en diferencias y las diferenciales, que era nuestro objetivo principal.

En el próximo artículo mostraremos las analogías anunciadas y estableceremos el llamado método de las diferencias finitas de resolución numérica de ecuaciones diferenciales, así como sus limitaciones. 